ANALYSIS AND MODELING OF LASER RAMPS AND SHOCKS IN TIITATIUM AND ZIRCONIUM WITH PHASE TRANSITIONS

O. Heuze, D. C. Swift

August 16, 2011

American Physical Society Topical Conference on Shock Compression of Condensed Matter Chicago, IL, United States June 26, 2011 through July 1, 2011 
This document was prepared as an account of work sponsored by an agency of the United States government. Neither the United States government nor Lawrence Livermore National Security, LLC, nor any of their employees makes any warranty, expressed or implied, or assumes any legal liability or responsibility for the accuracy, completeness, or usefulness of any information, apparatus, product, or process disclosed, or represents that its use would not infringe privately owned rights. Reference herein to any specific commercial product, process, or service by trade name, trademark, manufacturer, or otherwise does not necessarily constitute or imply its endorsement, recommendation, or favoring by the United States government or Lawrence Livermore National Security, LLC. The views and opinions of authors expressed herein do not necessarily state or reflect those of the United States government or Lawrence Livermore National Security, LLC, and shall not be used for advertising or product endorsement purposes. 


\title{
ANALYSIS AND MODELING OF LASER RAMPS AND SHOCKS IN TIITATIUM AND ZIRCONIUM WITH PHASE TRANSITIONS
}

\author{
Olivier Heuzé* and Damian C. Swift ${ }^{\dagger}$ \\ ${ }^{*}$ CEA/DIF, B.P. 12,91680 Bruyeres-Le Châtel, CEDEX, France \\ ${ }^{\dagger}$ Lawrence Livermore National Laboratory, 7000 East Avenue, Livermore, CA 94551, U.S.A.
}

\begin{abstract}
Using temporal pulse shaping, laser ablation can generate shocks or ramp loading in samples. Shock data can often be analyzed using analytic calculations, but hydrocode calculations are required in the case of ramps. When phase transitions occur, surface velocity histories may become much more complicated, requiring accurate hydrocode simulations for interpretation. The shock may be split by phase transitions and the slope of the ramp interacts with phase transition kinetics. The analysis of these experiments requires a good knowledge of phase transition thermodynamics i.e. an accurate multiphase equation of state (EOS). Recently, laser experiments have been performed on samples exhibiting phase transitions, complemented by a general model of multiphase EOS developed at CEA. The aim of the present study was to compare equilibrium multiphase EOS with qualitative and quantitative features of the experimental data. Multiphase EOS were constructed for Ti and $\mathrm{Zr}$ using static data. Good agreement was found between most experiments and calculations, demonstrating the accuracy of the multiphase EOS. In some cases, the experimental data show obvious kinetic effects.
\end{abstract}

Keywords: shock, plasticity, solid-solid transition

PACS: 47.40.+x, 62.20.F, 61.50.Ks, 64.70.K-

\section{INTRODUCTION}

The vast majority of equations of state (EOS) for dynamic loading simulations are a single-valued function of thermodynamic state. Phase changes, when represented explicitly rather than smeared out, are treated as instantaneous, and Maxwell constructions used to derive a single-valued EOS from multiple single-phase EOS. However, phase transitions are time dependent, and the transition state observed under dynamic loading is often different from the equilibrium EOS. For example, the $\alpha-\varepsilon$ transition in Fe occurs at $11 \mathrm{GPa}$ in static press experiments, and at $13 \mathrm{GPa}$ under dynamic loading on microsecond time scales [1].

Phase changes generally exhibit some degree of hysteresis, which is not captured using an equilibrium multiphase EOS. They may also depend on the elastic strain and plastic deformation states, which add to the free energy and impede recrystallization, respectively. Improved treatments should comprise a separate EOS for each phase, and transition rates. Theoretical EOS based on electronic structure can predict phase boundaries. Unfortunately, theoretical treatments accurate enough to come close to observed phase diagrams generally contain too many parameters for the phase boundaries to be adjusted.

We report the application of a semi-empirical method for constructing multiphase EOS to Ti and $\mathrm{Zr}$, and the comparison with shock and ramp loading. The single-phase EOS and representation of mixed phase regions have a form that is convenient for adding transition rates and contributions from elastic and plastic states. 


\section{MULTIPHASE EQUATION OF STATE}

For hydrocodes, the most convenient form for an EOS is pressure $p$ as a function of mass density $\rho$ (or specific volume $v=1 / \rho$ ) and specific internal energy $e$. Analytic EOS were used for each phase, and thermodynamic equilibrium was applied to obtain the phase diagram. Different phases are linked by integration constants: jumps in volume, entropy and internal energy. The single phase EOS were of the classical Mie-Grüneisen form expressed in $(v, e)$ form with $\Gamma / v$ constant, as has been used previously [2]:

$$
p(v, e)=p_{k}(v)+\Gamma_{0}\left\{\rho_{0} e_{0}[\varepsilon(v)-1]+e-e_{k}(v)\right\}
$$

where

$$
\varepsilon(v)=1+\Gamma_{0}\left(1-\rho_{0} v\right)
$$

and $e_{k}(v)$ is the cold compression curve, so $p_{k}=$ $-d e_{k} / d v$. Taking a constant specific heat capacity $c_{v}$, the temperature is

$$
T(v, e)=T_{0} \varepsilon(v)+\frac{e-e_{k}(v)}{c_{v}},
$$

giving a specific entropy

$$
s(v, e)=c_{v}\left\{\ln \left[\varepsilon(v)+\frac{e-e_{k}(v)}{c_{v} T_{0}}\right]+1-\varepsilon(v)\right\}+s_{0} .
$$

We have previously used a Birch-Murnaghan cold curve, but here we use a similar form that can be inverted much more efficently,

$$
p_{k}(v)=\frac{K_{0}}{N_{0}+1}\left[e^{\left(N_{0}+1\right) x}-1\right]
$$

where $x \equiv 1-\rho_{0} v$. Each single phase EOS is represented by eight parameters: $\rho_{0}, c_{v}, \Gamma_{0}, T_{0}$, the initial bulk modulus $K_{0}$, its derivative $N_{0}, e_{0}$, and $s_{0}$. For high pressure and high temperature phases, $\rho_{0}$ and $T_{0}$ are defined by convention such that $p\left(1 / \rho_{0}, c_{v} T_{0}\right)=$ 0 even if this state does not occur in reality. Electronic structure calculations can be used as well as experimental data, which is particularly useful when calibrating high pressure phases.

The boundaries of mixed phase regions can be represented accurately as polynomial parametric curves in $\rho-e$ space, allowing efficient determination of the equilibrium state. This approach has been tested for Sn ( 3 phases, 3 binary mixtures, and 1 triple point)

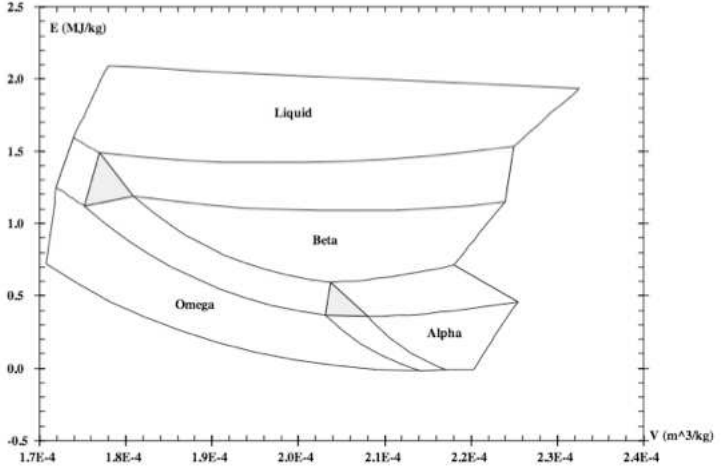

FIGURE 1. Multiphase EOS for Ti.

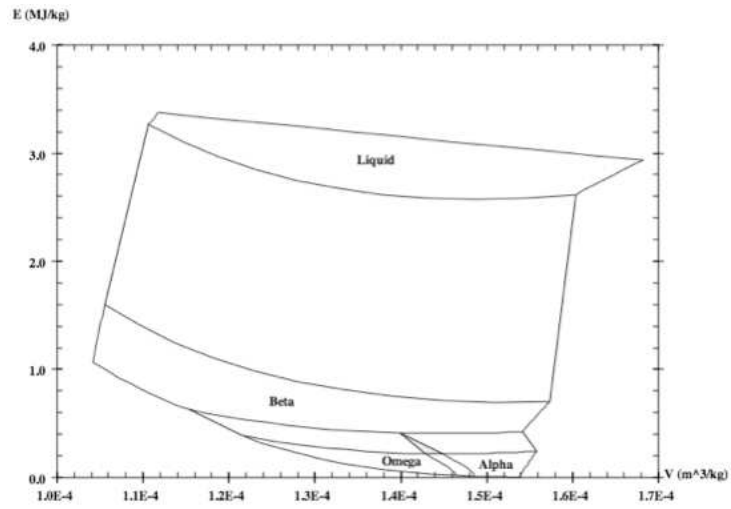

FIGURE 2. Multiphase EOS for Zr.

[3] and used to construct multiphase EOS for materials with complicated phase diagrams: $\mathrm{Bi}$ (10 phases, 19 binary mixtures, and 10 triple points) [4] and $\mathrm{H}_{2} \mathrm{O}$ (6 phases, 10 binary mixtures, and 7 triple points) [5].

In this work, multiphase EOS were constructed for Ti and Zr. The Ti EOS included 4 phases $(\alpha, \beta, \omega$, and liquid), 5 binary mixtures, and 2 triple points, and was consistent with the 2-phase EOS by Greeff et al [6] (Fig. 1). The Zr EOS also included 4 phases $(\alpha, \beta, \omega$, and liquid), then 4 binary mixtures and a single triple point, and is consistent with the 3-phase EOS by Greeff [7] (Fig. 2).

These EOS were analyzed to predict the pressure range over which different structures would appear on shock loading to different pressures, assuming instantaneous phase transtions and neglecting the effects of strength. For Ti, the $\alpha-\omega$ phase transition 
occurred near $2 \mathrm{GPa}$, and a double shock structure appeared between 2 and $8 \mathrm{GPa}$. For $\mathrm{Zr}$, the $\alpha-\omega$ transition occurred near $2 \mathrm{GPa}$ and the $\omega-\beta$ transition between 23 and $30 \mathrm{GPa}$, with double shocks between 3-8 and 23-35 GPa respectively.

\section{EXPERIMENTAL MEASUREMENTS}

Shock speed - particle speed measurements have been made for $\mathrm{Ti}$ and $\mathrm{Zr}$ [8] exhibiting discontinuities indicative of phase transitions. Similar results have been obtained from surface velocity histories in gas gun impact experiments [9, 10], and (on $\mathrm{Zr}$ ) from ramp loading at the $\mathrm{Z}$ machine [11]. These experiments used samples of order 1 millimeter thick, with microsecond time scales. Experiments have also been performed using laser ablation to induce shocks and ramps in rolled foil samples $25-60 \mu \mathrm{m}$ thick, at the Trident facility [12]. In these experiments, the sample was ablated directly and the velocity history of the opposite, free, surface was monitored using line-imaging Doppler velocimetry. As with the impact and $\mathrm{Z}$ experiments, inflections were evident in the velocity histories that suggested phase transformations. Some laser ablation experiments have been performed on $\mathrm{Ti}$ with in-situ x-ray diffraction, demonstrating changes to the crystal structure associated with the velocity features [13].

When performing hydrocode simulations of laser ablation experiments, it is desirable to use the laser irradiance history to predict the loading history applied to the sample. Radiation hydrodynamics simulations were used, with models previously found to be suitable for a wide range of elements and alloys $[14,15]$. The simulations for Ti produced pressures in reasonable agreement with the observed velocity histories, but the simulations for $\mathrm{Zr}$ produced pressures which were lower than observed by a factor $\sim 2$. For the purposes of comparison, the applied pressures in $\mathrm{Zr}$ were simply scaled. The radiation hydrodynamics simulations require a model for the Rosseland opacity of the ablated material, as well as an EOS valid in the dense plasma regime. Calculations of a simplified irradiance-pressure relation for each material gave a similarly different result for $\mathrm{Zr}$ than $\mathrm{Ti}$, including shell effects which, if occurring at the wrong irradiance, could account for the discrepancy.

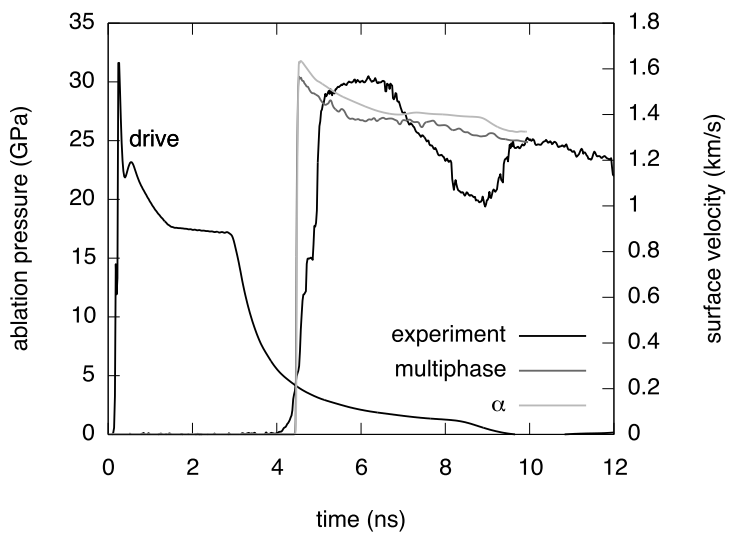

FIGURE 3. Example comparison between observed and simulated free surface velocity histories for Ti.

\section{Titanium}

For $\mathrm{Ti}$, microsecond scale experiments on commercially pure material indicated a phase transition, thought to be $\alpha-\omega$, starting at $9.7 \mathrm{GPa}$ [9], i.e. at a significantly higher pressure than the equilibrium phase diagram [6]. The nanosecond scale experiments, on material at least $99.99 \%$ pure, indicated the phase transition started around $11 \mathrm{GPa}$ with a shock and $10 \mathrm{GPa}$ with a ramp [12]. The multiphase simulations showed no double wave structure in the pressure range where it was observed experimentally, because the equilibrium phase change was so overdriven. There was little difference between simulations using the multiphase EOS and simulations used the $\alpha$ EOS only, as a limiting case of a slow phase transition. The shape of the peak of the shock was significantly different than the experiments, suggesting that the sound speed in the shocked state might be too high in the EOS at pressures $\sim 10-20 \mathrm{GPa}$. (Fig. 3.)

\section{Zirconium}

For $\mathrm{Zr}$, shock experiments on microsecond scales showed the onset of the $\alpha-\omega$ transition at $7.1 \mathrm{GPa}$ for very high purity material, increasing with impurity concentration $[9,10]$. The $\omega-\beta$ transition was not clear. For ramp loading on microsecond scales, the $\alpha-\omega$ transition showed a similar dependence on 


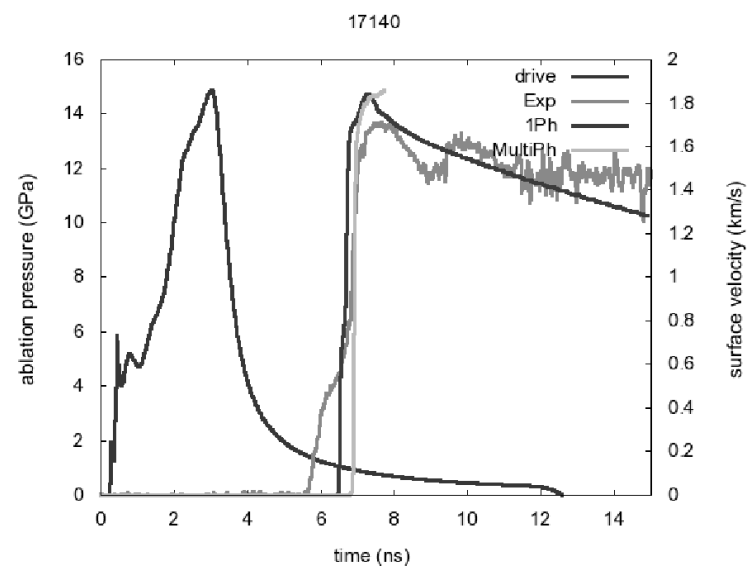

FIGURE 4. Example comparison between observed and simulated free surface velocity histories for $\mathrm{Zr}$.

purity, but the $\omega-\beta$ transition was clearly visible for all purities $[11,10]$. Nanosecond shock and ramp experiments on material of $99.8 \%$ purity - very low compared with the microsecond experiments - indicated phase transitions starting at 7 and $18 \mathrm{GPa}$ [12]. It is interesting that the $\alpha-\omega$ transition should be at a similar pressure as in the microsecond experiments at a purity that would point to a transition at a higher pressure. The higher elastic strain energy on these time scales acts to increase the free energy of the $\alpha$ phase; future work will assess whether this is sufficient to explain the difference. As with Ti, the velocity history in the region of the presumed $\alpha-\omega$ phase transition was not reproduced well using the equilibrium EOS or the $\alpha$ only EOS. The velocity history around the $\omega-\beta$ transition was in better agreement, as was the duration of the shock peak. (Fig. 4.)

\section{CONCLUSIONS}

Multiphase EOS were constructed for $\mathrm{Ti}$ and $\mathrm{Zr}$, using analytic single-phase EOS. Laser-driven shock and ramp data were obtained for $\mathrm{Ti}$ and $\mathrm{Zr}$. In Ti, the $\alpha-\omega$ transition appeared to occur $\sim 10 \mathrm{GPa}$, as was found on longer time scales. For comparison, the transition was at $\sim 2 \mathrm{GPa}$ for the equilibrium EOS. In $\mathrm{Zr}$, the $\alpha-\omega$ transition appeared $\sim 7 \mathrm{GPa}$, as for much purer material on microsecond scales. The $\omega-\beta$ transition appeared to starts at slightly lower pressure than for microsecond scales, which could be a finite strain effect. The equilibrium multi-phase EOS was not a good match to the velocity histories: kinetics will be added to be able to reproducing the experimental data.

\section{ACKNOWLEDGMENTS}

The authors would like to acknowledge the following people for their contributions to the experiments: Tom Tierney, Sheng-Nian Luo, Scott Greenfield (LANL); and Pedro Peralta (Arizona State University). The staff of the Trident facility contributed greatly to the experiments, in particular Randy Johnson, Sam Letzring, Ray Gonzales, Nathan Okamoto, Sha-Marie Reid, Danielle Pacheco, Tom Hurry, Fred Archuleta, and Tom Ortiz.

This project was performed under the CEA-NNSA collaborative program. The Livermore portion was performed under the auspices of the U.S. Department of Energy under contract DE-AC52-07NA27344[E LLNL

\section{REFERENCES}

1. Young, D.A., "Phase diagrams of the elements," University of California, 1990.

2. Horie, Y., and Duvall, G.E., in Proc. Army Symp. on Solid Mech., Baltimore, MD, Sep 10-11, 1968.

3. Missonnier, M. and Heuzé, O., AIP Conf. Proc. 845, 262, 2006.

4. Heuzé, O., AIP Conf. Proc. 845, 212, 2006.

5. Hebert, D., Bertron, I., and Heuzé, O., AIP Conf. Proc. 1195, 1205, 2009.

6. Greeff, C., Trinkle, D., and Albers, R., J. Appl. Phys. 90, 5, 2221, 2001.

7. Greeff, C., Mod. Simul. Mat. Sci. Eng. 13, 1015, 2005.

8. S.P. Marsh (Ed.), "LASL Shock Hugoniot Data", University of California (1980).

9. Gray III, G.T., et al, unpublished, 2004.

10. Rigg, P.A., Greeff, C.W., and Gray III, G.T., these proceedings.

11. Rigg, P., Knudson, M., and Hall, C., unpublished, 2004.

12. Swift, D.C., Tierney, T.E., and Luo, S.-N., unpublished, 2005.

13. McNaney, J.M., Lorenzana, H.E., et al, unpublished, 2006.

14. Swift, D.C., Tierney, T.E., Kopp, R.A., and Gammel, J.T., Phys. Rev. E, 69, 036406, 2004.

15. Swift, D.C., Gammel, J.T., and Clegg, S., Phys. Rev. E, 69, 056401, 2004. 